\title{
gs \\ Scaling law of coherent synchrotron radiation in a rectangular chamber
}

\author{
Yunhai Cai \\ SLAC National Accelerator Laboratory, Menlo Park, California 94025, USA
}

(Received 21 October 2013; published 12 February 2014)

\begin{abstract}
We study the threshold of longitudinal instability driven by coherent synchrotron radiation in a rectangular chamber in electron storage rings. Starting with Maxwell's equation, we first derive a point-charge wakefield for longitudinal motion. Then we use the wake, along with the Vlasov-Fokker-Planck equation, to show that the threshold can be described by a simple scaling law of $\xi=\xi^{\text {th }}(\chi, A, \hat{\beta})$, where $\xi$ is a dimensionless current, $\chi$ is the shielding parameter, $A$ is the aspect ratio of the vacuum chamber, and $\hat{\beta}$ is the damping rate relative to the rate of synchrotron oscillation. We further investigate the threshold with simulations for various values of aspect ratios. In particular, for a square chamber, we find that $\xi^{\text {th }} \approx 0.25$, which is a factor 2 lower than the threshold in free space.
\end{abstract}

DOI: 10.1103/PhysRevSTAB.17.020702

\section{INTRODUCTION}

Coherent synchrotron radiation (CSR) by circulating electrons in betatron was first investigated in an unpublished report [1] by Schwinger who was concerned with excessive radiation power. He discovered that the radiated power was actually suppressed by the parallel conducting walls. Essentially the same result was found later [2] by Nodvick and Saxon. Since then, mostly theoretical works $[3,4]$ continued along a path of calculating its wake and impedance.

A decade ago, an idea of utilizing CSR as coherent terahertz $(\mathrm{THz})$ radiation sources in the third generation light sources was systematically developed [5-8]. However, the brightness of these $\mathrm{THz}$ sources is severely limited by a longitudinal instability $[9,10]$ driven by CSR. The renewed experimental interests and diagnostics [11] provided by the radiation itself allows precision characterization of the threshold of this instability. Most recent observations [12-14] are in good agreement with a simple scaling law $[15,16]$, which is derived based on the parallelplate wake [4]. But why does the theory work so well even without sidewalls? What role if any do the sidewalls play? In particular, does the scaling law remain valid for a rectangular chamber? Those are the questions to be studied in this paper.

The earliest study of CSR in a rectangular chamber was carried out by Warnock and Morton [3] in the late 1990s. In particular, they derived the longitudinal impedance in terms

Published by the American Physical Society under the terms of the Creative Commons Attribution 3.0 License. Further distribution of this work must maintain attribution to the author(s) and the published article's title, journal citation, and DOI.
PACS numbers: 29.27.Bd, 41.75.Ht, 41.60.Ap, 02.30.Mv

of harmonic expansion by a rigid bunch, sometimes called a needle beam with a small vertical height. Recently, the investigation has been extended to include the transient effect [17-19] under the paraxial approximation. In this paper, we will continue these works with a focus on the wakefield and impedance generated by a point charge and its scaling property similar to those found by Murphy, Krinsky, and Gluckstern [4] in the parallel plate model.

In Secs. II and III, we will solve Maxwell's equation with the boundary condition on a rectangular chamber made with perfectly conducting medal. Continuing in Sec. IV, we will introduce the longitudinal wakefield and impedance naturally according to the functional form in the derived solution. In Sec. V, the scaling property of the impedance will be derived under an approximation that is suitable for most circular accelerators. In Sec. VI, to validate our point-charge wakefield, we will derive the bunch wakes and compare them with the previously known results. In Sec. VII, we will introduce the equation that governs the longitudinal beam dynamics and derive a functional form of the scaling law for the threshold of instability. To determine the function itself, we will perform simulations for chambers with various aspect ratios in Sec. VIII. Finally, in Sec. IX, we will make some concluding remarks.

\section{FIELD EQUATIONS}

In this and the next two sections, we will closely follow the approach used by Warnock and Morton [3] to derive the electromagnetic field and impedance for a point charge circulating in a rectangular chamber. We will start with the Maxwell equation in its component form. In cylindrical coordinates, $r, \theta, y$, they can be written as 


$$
\begin{gathered}
\frac{1}{r} \frac{\partial\left(r E_{r}\right)}{\partial r}+\frac{1}{r} \frac{\partial E_{\theta}}{\partial \theta}+\frac{\partial E_{y}}{\partial y}=4 \pi \rho, \\
\frac{1}{r} \frac{\partial B_{y}}{\partial \theta}-\frac{\partial B_{\theta}}{\partial y}-\frac{1}{c} \frac{\partial E_{r}}{\partial t}=\frac{4 \pi}{c} j_{r}, \\
\frac{\partial B_{r}}{\partial y}-\frac{\partial B_{y}}{\partial r}-\frac{1}{c} \frac{\partial E_{\theta}}{\partial t}=\frac{4 \pi}{c} j_{\theta}, \\
\frac{1}{r} \frac{\partial\left(r B_{\theta}\right)}{\partial r}-\frac{1}{r} \frac{\partial B_{r}}{\partial \theta}-\frac{1}{c} \frac{\partial E_{y}}{\partial t}=\frac{4 \pi}{c} j_{y}, \\
\frac{1}{r} \frac{\partial\left(r B_{r}\right)}{\partial r}+\frac{1}{r} \frac{\partial B_{\theta}}{\partial \theta}+\frac{\partial B_{y}}{\partial y}=0, \\
\frac{1}{r} \frac{\partial E_{y}}{\partial \theta}-\frac{\partial E_{\theta}}{\partial y}+\frac{1}{c} \frac{\partial B_{r}}{\partial t}=0, \\
\frac{\partial E_{r}}{\partial y}-\frac{\partial E_{y}}{\partial r}+\frac{1}{c} \frac{\partial B_{\theta}}{\partial t}=0, \\
\frac{1}{r} \frac{\partial\left(r E_{\theta}\right)}{\partial r}-\frac{1}{r} \frac{\partial E_{r}}{\partial \theta}+\frac{1}{c} \frac{\partial B_{y}}{\partial t}=0,
\end{gathered}
$$

To solve these equations, we make Fourier expansions:

$$
\begin{aligned}
E_{r}(r, \theta, y, t)= & \int_{-\infty}^{\infty} d \omega e^{-i \omega t} \sum_{n=-\infty}^{\infty} e^{i n \theta} \sum_{p=1}^{\infty} \sin \alpha_{p}\left(y+\frac{h}{2}\right) \\
& \times E_{r n p}(r, \omega), \\
E_{\theta}(r, \theta, y, t)= & \int_{-\infty}^{\infty} d \omega e^{-i \omega t} \sum_{n=-\infty}^{\infty} e^{i n \theta} \sum_{p=1}^{\infty} \sin \alpha_{p}\left(y+\frac{h}{2}\right) \\
& \times E_{\theta n p}(r, \omega), \\
B_{y}(r, \theta, y, t)= & \int_{-\infty}^{\infty} d \omega e^{-i \omega t} \sum_{n=-\infty}^{\infty} e^{i n \theta} \sum_{p=1}^{\infty} \sin \alpha_{p}\left(y+\frac{h}{2}\right) \\
& \times B_{y n p}(r, \omega) .
\end{aligned}
$$

Here we consider only a perfectly conducting chamber as illustrated in Fig. 1. To satisfy the boundary conditions, $E_{r}(r, \theta, \pm h / 2, t)=E_{\theta}(r, \theta, \pm h / 2, t)=0$, and $B_{y}(r, \theta, \pm h / 2, t)=0$, we set the wave number in the vertical direction

$$
\alpha_{p}=\frac{p \pi}{h},
$$

where $h$ is the height of the rectangular chamber. For the other field components and sources, we choose their expansion:

$$
\begin{gathered}
\left(E_{y}, B_{r}, B_{\theta}\right) \sim(\cos , \cos , \cos ), \\
\left(j_{r}, j_{\theta}, j_{y}, \rho\right) \sim(\sin , \sin , \cos , \sin ),
\end{gathered}
$$

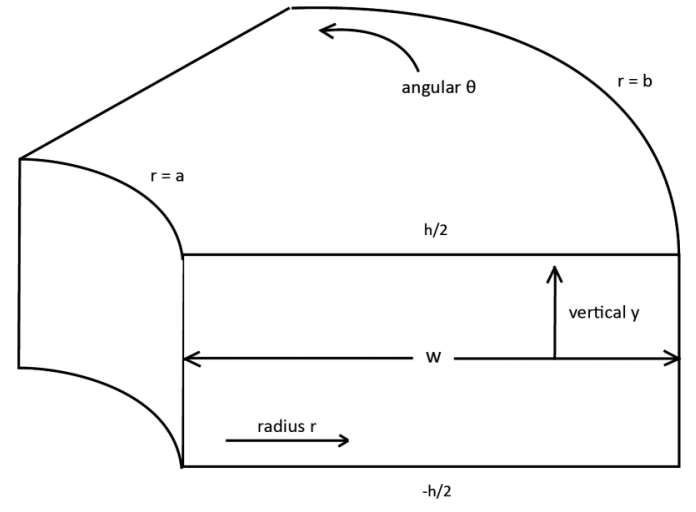

FIG. 1. A segment of a rectangular vacuum chamber in the cylindrical coordinate.

accordingly so that each component equation in Eq. (2.1) has either a sine or cosine dependence of $\alpha_{p}(y+h / 2)$.

Solving $E_{r n p}, B_{\theta n p}, B_{r n p}, E_{\theta n p}$ according to $B_{y n p}, E_{y n p}$ and charge sources, we obtain

$$
\begin{aligned}
& E_{r n p}=\frac{-1}{\gamma_{p}^{2}}\left[\alpha_{p} \frac{\partial E_{y n p}}{\partial r}+\frac{\omega}{c}\left(\frac{n}{r} B_{y n p}+i \frac{4 \pi}{c} j_{r n p}\right)\right], \\
& B_{\theta n p}=\frac{i}{\gamma_{p}^{2}}\left[\frac{\omega}{c} \frac{\partial E_{y n p}}{\partial r}+\alpha_{p}\left(\frac{n}{r} B_{y n p}+i \frac{4 \pi}{c} j_{r n p}\right)\right], \\
& E_{\theta n p}=\frac{-i}{\gamma_{p}^{2}}\left[\alpha_{p} \frac{n}{r} E_{y n p}+\frac{\omega}{c}\left(\frac{\partial B_{y n p}}{\partial r}+\frac{4 \pi}{c} j_{\theta n p}\right)\right],
\end{aligned}
$$

$B_{r n p}=\frac{1}{\gamma_{p}^{2}}\left[\frac{\omega n}{c} \frac{n}{r} E_{y n p}+\alpha_{p}\left(\frac{\partial B_{y n p}}{\partial r}+\frac{4 \pi}{c} j_{\theta n p}\right)\right]$,

where $\gamma_{p}$ is defined by

$$
\gamma_{p}^{2}=\left(\frac{\omega}{c}\right)^{2}-\alpha_{p}^{2}
$$

The vertical components of the fields $E_{y n p}$ and $B_{y n p}$ satisfy

$$
\begin{gathered}
\frac{1}{r} \frac{\partial}{\partial r}\left(r \frac{\partial E_{y n p}}{\partial r}\right)+\left(\gamma_{p}^{2}-\frac{n^{2}}{r^{2}}\right) E_{y n p} \\
\quad=\frac{4 \pi}{c}\left(-i \frac{\omega}{c} j_{y n p}+\alpha_{p} c \rho_{n p}\right),
\end{gathered}
$$




$$
\begin{aligned}
& \frac{1}{r} \frac{\partial}{\partial r}\left(r \frac{\partial B_{y n p}}{\partial r}\right)+\left(\gamma_{p}^{2}-\frac{n^{2}}{r^{2}}\right) B_{y n p} \\
& =\frac{4 \pi}{c}\left[-\frac{1}{r} \frac{\partial}{\partial r}\left(r j_{\theta n p}\right)+i \frac{n}{r} j_{r n p}\right] .
\end{aligned}
$$

Without the sources $\rho, j$, the general solution of these two equations is a linear combination of the Bessel functions $J_{n}\left(\gamma_{p} r\right)$ and $Y_{n}\left(\gamma_{p} r\right)$.

\section{FIELD SOLUTIONS}

With the sources, we need to solve the equation,

$$
\frac{1}{r} \frac{\partial}{\partial r}\left(r \frac{\partial \psi_{n}}{\partial r}\right)+\left(\gamma_{p}^{2}-\frac{n^{2}}{r^{2}}\right) \psi_{n}=v(r)
$$

where $v$ is the inhomogeneous term. A particular solution is given by [20]

$$
\begin{aligned}
\psi_{n}(r)= & y_{2}(r) \int_{a}^{r} \frac{v(u)}{\Delta\left(y_{1}, y_{2}\right)} y_{1}(u) d u \\
& +y_{1}(r) \int_{r}^{b} \frac{v(u)}{\Delta\left(y_{1}, y_{2}\right)} y_{2}(u) d u,
\end{aligned}
$$

where $y_{1}$ and $y_{2}$ are two independent solutions of the homogeneous equation,

$$
\frac{1}{r} \frac{\partial}{\partial r}\left(r \frac{\partial \psi_{n}}{\partial r}\right)+\left(\gamma_{p}^{2}-\frac{n^{2}}{r^{2}}\right) \psi_{n}=0,
$$

and $\Delta\left(y_{1}, y_{2}\right)$ is the Wronskian

$$
\Delta\left(y_{1}, y_{2}\right)=\left|\begin{array}{ll}
y_{1} & y_{1}^{\prime} \\
y_{2} & y_{2}^{\prime}
\end{array}\right|
$$

It is worth noting that we have chosen the homogeneous solution so that $\psi_{n}(a)=y_{1}(a)$ and $\psi_{n}(b)=y_{2}(b)$. It is therefore convenient to choose $y_{1}$ and $y_{2}$ so that they satisfy the boundary condition at $r=a$ and $r=b$, respectively.

For a charged particle that orbits on a circle with radius of $R$ in the plane defined by $y=0$, its charge density is given in terms of delta functions,

$$
\rho(r, \theta, y, t)=\frac{q}{r} \delta_{p}\left(\theta-\omega_{0} t\right) \delta(y) \delta(r-R),
$$

where $\delta_{p}(\theta)$ should be understood as a periodic delta function and its angular frequency $\omega_{0}=\beta c / R$. Similarly, the current density can be written as

$$
\left(j_{r}, j_{\theta}, j_{z}\right)=(0, \beta c \rho, 0)
$$

In the Fourier space, we have

$$
\begin{gathered}
\rho_{n p}(r, \omega)=\frac{q}{\pi r h} \sin \frac{\pi p}{2} \delta\left(\omega-n \omega_{0}\right) \delta(r-R), \\
j_{\theta n p}(r, \omega)=\beta c \rho_{n p}(r, \omega) .
\end{gathered}
$$

Note that these source terms are zero if $p$ is an even number. So only odd $p$ modes are excited by this source in the $y=0$ plane.

Referring to Eqs. (2.13) and (3.1) for $E_{y n p}$, we have $v=4 \pi \alpha_{p} \rho_{n p}$. To satisfy the boundary condition, $E_{y n p}(a)=E_{y n p}(b)=0$, we choose $y_{1}=p_{n}\left(\gamma_{p} r, \gamma_{p} a\right)$ and $y_{2}=p_{n}\left(\gamma_{p} r, \gamma_{p} b\right)$. Here we use the notation of the Bessel function cross products,

$$
\begin{aligned}
& p_{n}(x, y)=J_{n}(x) Y_{n}(y)-Y_{n}(x) J_{n}(y), \\
& q_{n}(x, y)=J_{n}(x) Y_{n}^{\prime}(y)-Y_{n}(x) J_{n}^{\prime}(y), \\
& r_{n}(x, y)=J_{n}^{\prime}(x) Y_{n}(y)-Y_{n}^{\prime}(x) J_{n}(y), \\
& s_{n}(x, y)=J_{n}^{\prime}(x) Y_{n}^{\prime}(y)-Y_{n}^{\prime}(x) J_{n}^{\prime}(y) .
\end{aligned}
$$

Carrying out two integrals in Eq. (3.2), we obtain

$$
\begin{aligned}
E_{y n p}(r, \omega)= & \frac{2 \pi^{2} \alpha_{p} \Phi}{p_{n}\left(\gamma_{p} a, \gamma_{p} b\right)} \\
& \times \begin{cases}p_{n}\left(\gamma_{p} r, \gamma_{p} a\right) p_{n}\left(\gamma_{p} R, \gamma_{p} b\right) & \text { if } r \leq R \\
p_{n}\left(\gamma_{p} r, \gamma_{p} b\right) p_{n}\left(\gamma_{p} R, \gamma_{p} a\right) & \text { if } r \geq R,\end{cases}
\end{aligned}
$$

where

$$
\Phi=\frac{q}{\pi h} \sin \frac{\pi p}{2} \delta\left(\omega-n \omega_{0}\right) .
$$

Similarly, for $B_{y n p}$, we have

$$
v=-\frac{4 \pi}{c r} \frac{\partial\left(r j_{\theta n p}\right)}{\partial r}
$$

with $B_{y n p}^{\prime}(a)=B_{y n p}^{\prime}(b)=0$. Here, we choose $y_{1}=$ $q_{n}\left(\gamma_{p} r, \gamma_{p} a\right)$ and $y_{2}=q_{n}\left(\gamma_{p} r, \gamma_{p} b\right)$. Again carrying out the integrals, we find

$$
\begin{aligned}
B_{y n p}(r, \omega)= & \frac{2 \pi^{2} \beta \gamma_{p} \Phi}{s_{n}\left(\gamma_{p} a, \gamma_{p} b\right)} \\
& \times \begin{cases}q_{n}\left(\gamma_{p} r, \gamma_{p} a\right) s_{n}\left(\gamma_{p} R, \gamma_{p} b\right) & \text { if } r \leq R \\
q_{n}\left(\gamma_{p} r, \gamma_{p} b\right) s_{n}\left(\gamma_{p} R, \gamma_{p} a\right) & \text { if } r \geq R .\end{cases}
\end{aligned}
$$

With the solutions of $E_{y n p}$ and $B_{y n p}$ in hand, we can compute all components of electric and magnetic fields. In 
particular, we can calculate the electric field along the circular orbit and find

$$
\begin{aligned}
E_{\theta}(R, \theta, 0, t)= & -\frac{2 \pi i q}{h R} \sum_{n=-\infty}^{\infty} n e^{i n\left(\theta-\omega_{0} t\right)} \\
& \times \sum_{p(\text { odd }) \geq 1}^{\infty}\left[\beta^{2} \frac{s_{n}\left(\gamma_{p} R, \gamma_{p} a\right) s_{n}\left(\gamma_{p} R, \gamma_{p} b\right)}{s_{n}\left(\gamma_{p} a, \gamma_{p} b\right)}\right. \\
& \left.+\left(\frac{\alpha_{p}}{\gamma_{p}}\right)^{2} \frac{p_{n}\left(\gamma_{p} R, \gamma_{p} a\right) p_{n}\left(\gamma_{p} R, \gamma_{p} b\right)}{p_{n}\left(\gamma_{p} a, \gamma_{p} b\right)}\right],
\end{aligned}
$$

where we have $\omega=n \omega_{0}$ as a consequence of the integration of $\omega$. Now, $\gamma_{p}$ is given by

$$
\gamma_{p}=\sqrt{\left(\frac{n \beta}{R}\right)^{2}-\alpha_{p}^{2}} .
$$

\section{WAKEFIELD AND IMPEDANCE}

It is obvious that the wavelength of the Fourier mode $n$ is $\lambda_{n}=2 \pi R / n$. Hence its wave number $k_{n}=2 \pi / \lambda_{n}=n / R$. To study beam dynamics of a single bunch with length $\sigma_{z}$, we are interested in the modes with $\lambda_{n}<2 \pi \sigma_{z}$ or equivalently $k_{n}>1 / \sigma_{z}$. This implies $n>R / \sigma_{z}$. For a typical storage ring, say $R=10$ meter and $\sigma_{z}=5 \mathrm{~mm}$, we have $n>2000$. In this region of parameters, $k_{n}=1 / R$ is nearly continuous in comparison to $1 / \sigma_{z}$ and therefore we can approximate the summation of $n$ :

$$
\sum_{n=-\infty}^{\infty} \rightarrow R \int_{-\infty}^{\infty} d k
$$

by a continuous integral of $k=n / R$. Now the longitudinal field for CSR can be written as

$$
\begin{aligned}
E_{\theta}(z)= & -\frac{2 \pi i q}{h} \int_{-\infty}^{\infty} d k n e^{i k z} \\
& \times \sum_{p(\text { odd }) \geq 1}^{n \beta h / \pi R}\left[\beta^{2} \frac{s_{n}\left(\gamma_{p} R, \gamma_{p} a\right) s_{n}\left(\gamma_{p} R, \gamma_{p} b\right)}{s_{n}\left(\gamma_{p} a, \gamma_{p} b\right)}\right. \\
& \left.+\left(\frac{\alpha_{p}}{\gamma_{p}}\right)^{2} \frac{p_{n}\left(\gamma_{p} R, \gamma_{p} a\right) p_{n}\left(\gamma_{p} R, \gamma_{p} b\right)}{p_{n}\left(\gamma_{p} a, \gamma_{p} b\right)}\right],
\end{aligned}
$$

where $z=s-\beta c t$ describes the circular position relative to the point charge, where the positive $z$ points toward the front in the direction of motion. Here we have restricted the summation to the modes that are propagating and have real $\gamma_{p}$. Now we have shown that the dependencies of $s$ and $t$ are through $z$ and therefore naturally we shall introduce a concept of wakefield [21] integrated over $2 \pi R$,

$$
W(z)=-2 \pi R E_{\theta}(z) / q .
$$

Now if we use the concept of the impedance,

$$
W(z)=\frac{\beta c}{2 \pi} \int_{-\infty}^{\infty} d k e^{i k z} Z(k)
$$

we can derive the impedance $Z(k)$ and then write it in terms of

$$
\begin{aligned}
\frac{Z(n)}{n}= & \frac{2 \pi^{2} i R}{\beta h}\left(\frac{4 \pi}{c}\right) \\
& \times \sum_{p(\text { odd }) \geq 1}^{n \beta h / \pi R}\left[\beta^{2} \frac{s_{n}\left(\gamma_{p} R, \gamma_{p} a\right) s_{n}\left(\gamma_{p} R, \gamma_{p} b\right)}{s_{n}\left(\gamma_{p} a, \gamma_{p} b\right)}\right. \\
& \left.+\left(\frac{\alpha_{p}}{\gamma_{p}}\right)^{2} \frac{p_{n}\left(\gamma_{p} R, \gamma_{p} a\right) p_{n}\left(\gamma_{p} R, \gamma_{p} b\right)}{p_{n}\left(\gamma_{p} a, \gamma_{p} b\right)}\right],
\end{aligned}
$$

to compare the result obtained by Warnock and Morton [3]. The results agree if we set $\Lambda_{p}=2, Z_{0}=4 \pi / c, \omega=n \omega_{0}$, and add the finite upper bound of the summation in Eq. (3.9) in their paper. It is worth pointing out that we have used a point charge instead of a charged bunch in the previous work [3] as the source for generating the field.

\section{SCALING PROPERTY}

For simplicity, we will assume that $\beta=1$ and the beam is at the center of the chamber. As we have discussed at the beginning of this section, we are dealing with a very large Fourier mode number $n$. For such large $n$, it is well known that asymptotic expansions are an excellent way to calculate the values of the Bessel functions. In particular, we need to compute the Bessel functions in the form of $J_{n}\left(n \sqrt{1-x^{2}}\right)$, which leads us to the uniform asymptotic expansion [22] associated with the Airy function Ai. Applying the first term in the expansions of the Bessel functions and their derivatives, namely,

$$
\begin{gathered}
J_{n}\left(n \sqrt{1-x^{2}}\right) \sim\left(\frac{2}{n}\right)^{1 / 3} \mathrm{Ai}\left[\left(\frac{n}{2}\right)^{2 / 3} x^{2}\right], \\
Y_{n}\left(n \sqrt{1-x^{2}}\right) \sim-\left(\frac{2}{n}\right)^{1 / 3} \mathrm{Bi}\left[\left(\frac{n}{2}\right)^{2 / 3} x^{2}\right], \\
J_{n}^{\prime}\left(n \sqrt{1-x^{2}}\right) \sim-\frac{1}{\sqrt{1-x^{2}}}\left(\frac{2}{n}\right)^{2 / 3} \mathrm{Ai}^{\prime}\left[\left(\frac{n}{2}\right)^{2 / 3} x^{2}\right], \\
Y_{n}^{\prime}\left(n \sqrt{1-x^{2}}\right) \sim \frac{1}{\sqrt{1-x^{2}}}\left(\frac{2}{n}\right)^{2 / 3} \mathrm{Bi}^{\prime}\left[\left(\frac{n}{2}\right)^{2 / 3} x^{2}\right],
\end{gathered}
$$

to Eq. (4.5), we can express the impedance in terms of Airy functions, 


$$
\begin{aligned}
\frac{Z(n)}{n}= & -\frac{2 \pi^{2} i h}{R}\left(\frac{4 \pi}{c}\right)\left(\frac{\hat{k}}{2}\right)^{-4 / 3} \sum_{p(\text { odd }) \geq 1}^{k h / \pi}\left[\frac{\hat{s}\left(u, u^{+}\right) \hat{s}\left(u, u^{-}\right)}{\hat{s}\left(u^{+}, u^{-}\right)}\right. \\
& \left.+u \frac{\hat{p}\left(u, u^{+}\right) \hat{p}\left(u, u^{-}\right)}{\hat{p}\left(u^{+}, u^{-}\right)}\right]
\end{aligned}
$$

where $\hat{k}$ is a dimensionless wave number,

$$
\hat{k}=k h^{3 / 2} / R^{1 / 2}
$$

and $\hat{p}$ and $\hat{s}$ are the cross products of Airy functions and their derivatives,

$$
\begin{gathered}
\hat{p}(x, y)=\operatorname{Ai}(x) \operatorname{Bi}(y)-\operatorname{Bi}(x) \operatorname{Ai}(y), \\
\hat{s}(x, y)=\operatorname{Ai}^{\prime}(x) \operatorname{Bi}^{\prime}(y)-\operatorname{Bi}^{\prime}(x) \operatorname{Ai}^{\prime}(y) .
\end{gathered}
$$

Their arguments $u$ and $u^{ \pm}$can be computed by applying $\gamma_{p}$ from Eq. (3.18),

$$
\begin{gathered}
u=\frac{\pi^{2} p^{2}}{2^{2 / 3}} \hat{k}^{-4 / 3}, \\
u^{ \pm}=\frac{\pi^{2} p^{2}}{2^{2 / 3}} \hat{k}^{-4 / 3} \pm \frac{A}{2^{2 / 3}} \hat{k}^{2 / 3},
\end{gathered}
$$

where $A=w / h$ is the aspect ratio and $w=b-a$ the width of the rectangular chamber. We should mention that it is necessary to assume that $w / R \ll 1$ and $p \ll k h / \pi$ in our derivation. The first approximation is well justified for typical accelerators and the second one implies that $p$ should not be too large in the expansion. As we will see later, one needs to expand $p$ up to 7 in the cases of interests when $1 \leq A \leq 3$.

It is worth emphasizing that all dependency of the wave number $k$ is through $\hat{k}$. This scaling property of the impedance was first discovered by Murphy, Krinsky, and Gluckstern for parallel plates. Here we have shown that it remains valid for a rectangular chamber. Of course, an additional parameter, namely the aspect ratio $A$, is necessary to describe the impedance. We should mention that our impedance agrees with the expression derived by Agoh [18] under the paraxial approximation if we set $\beta=1$, $\Lambda_{p}=1, Z_{0}=4 \pi / c$, add the finite upper bound, and finally multiply a factor of $2 \pi R$ in Eq. (69) in his paper.

Given the impedance in Eq. (5.5), it is more convenient to calculate first the integral of the wake $S(z)$ by

$$
S(z)=\frac{c}{2 \pi i} \int_{-\infty}^{\infty} d k e^{i k z}\left(\frac{Z(k)}{k}\right),
$$

and then compute the wake with

$$
W(z)=\frac{d S(z)}{d z} .
$$

The integral in Eq. (5.11) can be carried out by applying the calculus of residues in the complex plane of $k$. The contour is chosen to be slightly above the real axis along the positive direction, circling back on a huge half circle either on the upper plane for positive $z$ or the lower one for negative $z$. As a result, the wake generated by the point charge at $z=0$ can expressed as a summation,

$$
W(\hat{z})=\frac{R}{h^{2}} \sum_{j} \hat{k}_{j} \frac{R_{s}}{Q}\left(\hat{k}_{j}\right)\left[\Theta(-\hat{z}) \cos \left(\hat{k}_{j} \hat{z}\right)-\frac{\operatorname{sgn}(\hat{z})}{2} e^{-\hat{k}_{j}|\hat{z}|}\right],
$$

where $\Theta$ is the step function and sgn is for the sign function. We note $\hat{z}=z R^{1 / 2} / h^{3 / 2}$ as a dimensionless longitudinal position and have assumed $\hat{k}_{j}>0$. The poles are defined by zeros of $\hat{s}\left(u^{+}, u^{-}\right)$or $\hat{p}\left(u^{+}, u^{-}\right)$. For each index $j$, we find four poles at $\pm \hat{k}_{j}, \pm i \hat{k}_{j}$. The poles on the real axis contribute to the oscillations behind the charge and imaginary ones lead to the exponential decays. Here we plot the wake potential as a function of the dimensionless position $\hat{z}$ in Fig. 2 for aspect ratio $A=1$. The rapid damping immediately behind the driving particle is due to the decoherence among high harmonics. Afterwards, it recoheres partially at the period of the lowest mode.

For each index $j$, the oscillation term can be interpreted as the resonant wake driven by a parallel inductanceresistance-capacitor circuit with an infinite quality factor $Q$. In such resonance, the quantity $R_{s} / Q$ remains finite and meaningful. The additional damped terms are presumably due to the circular geometry. A hint is coming from the fact that it has contributed to the wake ahead $(\hat{z}>0)$ as shown in Fig. 2. This is possible because the radiated photons can take a short cut in a straight line getting ahead of the charged particle that is orbiting on a circle.

The roots of $\hat{s}$ can be easily found with a numerical search for a given $p$. With the root $\hat{k}_{j}$, the dimensionless $R_{s} / Q$ can be computed with

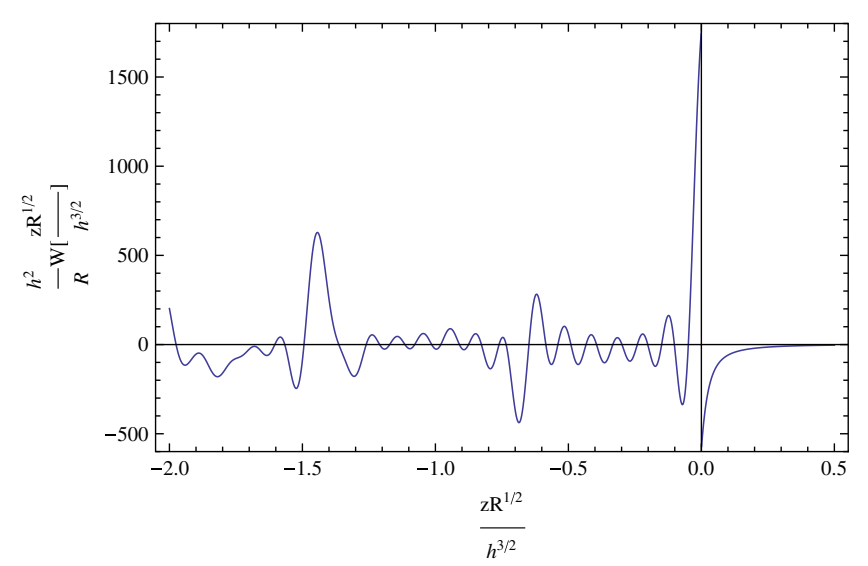

FIG. 2 (color online). A point-charge wakefield as a function of the dimensionless longitudinal position $\hat{z}$ for a square chamber. 


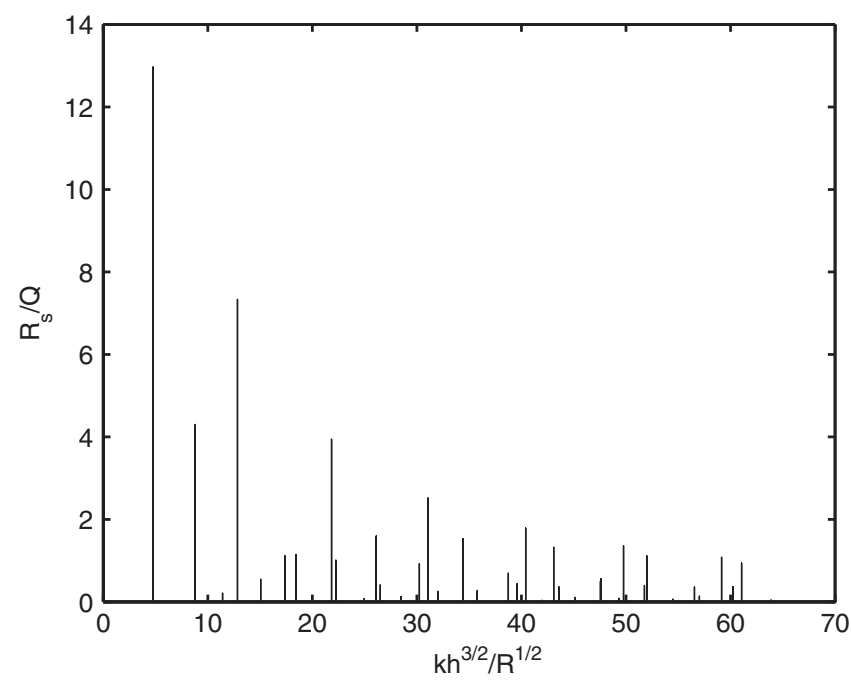

FIG. 3. A dimensionless $R_{s} / Q$ for the resonances as a function of the dimensionless wave number $\hat{k}$ for a square chamber.

$$
\frac{R_{s}}{Q}\left(\hat{k}_{j}\right)=-\Upsilon \hat{k}_{j}^{-4 / 3} \frac{\hat{s}\left(u_{j}, u_{j}^{+}\right) \hat{s}\left(u_{j}, u_{j}^{-}\right)}{d \hat{s}\left(u_{j}^{+}, u_{j}^{-}\right) / d \hat{k}}
$$

where $\Upsilon=32 \pi^{3} 2^{1 / 3}$. Similarly, for the $\hat{p}$ roots,

$$
\frac{R_{s}}{Q}\left(\hat{k}_{j}\right)=-\Upsilon \hat{k}_{j}^{-4 / 3} u_{j} \frac{\hat{p}\left(u_{j}, u_{j}^{+}\right) \hat{p}\left(u_{j}, u_{j}^{-}\right)}{d \hat{p}\left(u_{j}^{+}, u_{j}^{-}\right) / d \hat{k}} .
$$

The wake has no explicit dependence on the aspect ratio $A$. But its effect is hidden in the values of the roots $\hat{k}_{j}$ through Eq. (5.10). For a square chamber $A=1$, the dimensionless $R_{s} / Q$ is shown in Fig. 3 with 46 modes, $p \leq 7$, and $\hat{k}<65$. For two rectangular chambers with $A=2$ and $A=3$, we show their resonance spectra in Fig. 4 with $p \leq 5$. Naturally, the larger the aspect ratio the more resonances but with reducing strengths. The shape of their envelopes is very similar in these two cases.

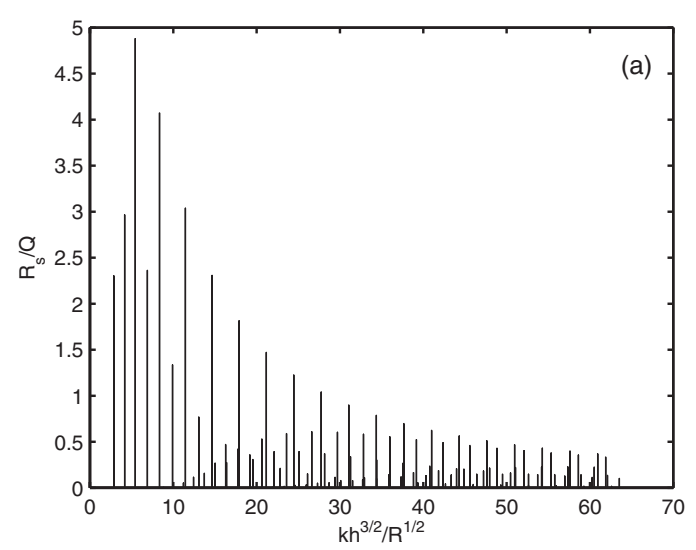

\section{BUNCH WAKE}

Sometimes, it is useful to have a wakefield driven by a Gaussian bunch with length $\sigma_{z}$. Given the point-charge wake $W(\hat{z})$, the bunch wake can be computed by a convolution to its distribution,

$$
W_{\chi}(\hat{z})=\frac{1}{\sqrt{2 \pi} \chi} \int_{-\infty}^{\infty} d \zeta W(\hat{z}-\zeta) e^{-\frac{\zeta^{2}}{2 \chi^{2}}}
$$

where we introduce a shielding parameter which is defined as

$$
\chi=\sigma_{z} R^{1 / 2} / h^{3 / 2}
$$

Carrying out the integral, we find

$$
W_{\chi}(\hat{z})=\frac{R}{h^{2}} \sum_{j} \hat{k}_{j} \frac{R_{s}}{Q}\left(\hat{k}_{j}\right)\left[w_{\chi o}\left(\hat{k}_{j}, \hat{z}\right)+w_{\chi d}\left(\hat{k}_{j}, \hat{z}\right)\right],
$$

where

$$
\begin{aligned}
w_{\chi o}(\hat{k}, \hat{z})= & \frac{e^{-\frac{1}{2} \hat{k}\left(2 i \hat{z}+\hat{k} \chi^{2}\right)}}{4}\left[\operatorname{erfc}\left(\frac{\hat{z}-i \hat{k} \chi^{2}}{\chi \sqrt{2}}\right)\right. \\
& \left.+e^{2 i \hat{k} \hat{z}} \operatorname{erfc}\left(\frac{\hat{z}+i \hat{k} \chi^{2}}{\chi \sqrt{2}}\right)\right],
\end{aligned}
$$

$$
\begin{aligned}
w_{\chi d}(\hat{k}, \hat{z})= & \frac{e^{\frac{1}{2} \hat{k}\left(-2 \hat{z}+\hat{k} \chi^{2}\right)}}{4}\left[\operatorname{erfc}\left(\frac{\hat{z}-\hat{k} \chi^{2}}{\chi \sqrt{2}}\right)\right. \\
& \left.+e^{2 \hat{k} \hat{z}} \operatorname{erfc}\left(\frac{\hat{z}+\hat{k} \chi^{2}}{\chi \sqrt{2}}\right)-2\right],
\end{aligned}
$$

in terms of the complementary error function erfc. Here we use the subscript " $o$ " to indicate its origin from the oscillation terms and " $d$ " for the damped ones.

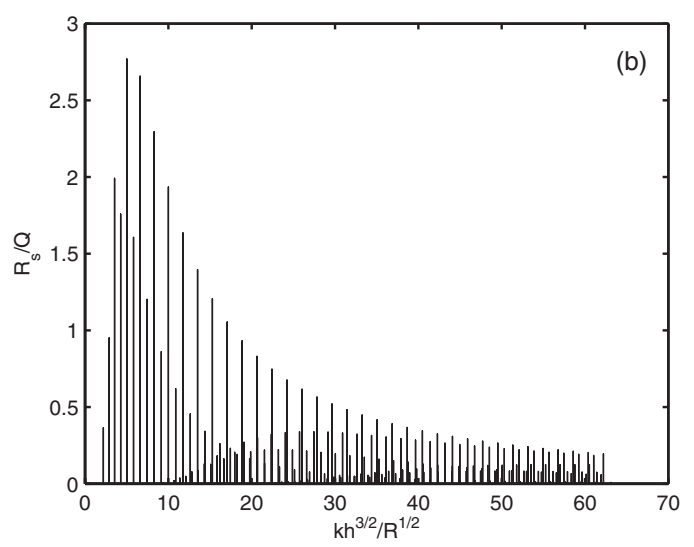

FIG. 4. $R_{s} / Q$ for the resonances as a function of $\hat{k}$ for rectangular chamber with an aspect ratio (a) $A=2$ with 112 resonances and (b) $A=3$ with 204 resonances. 

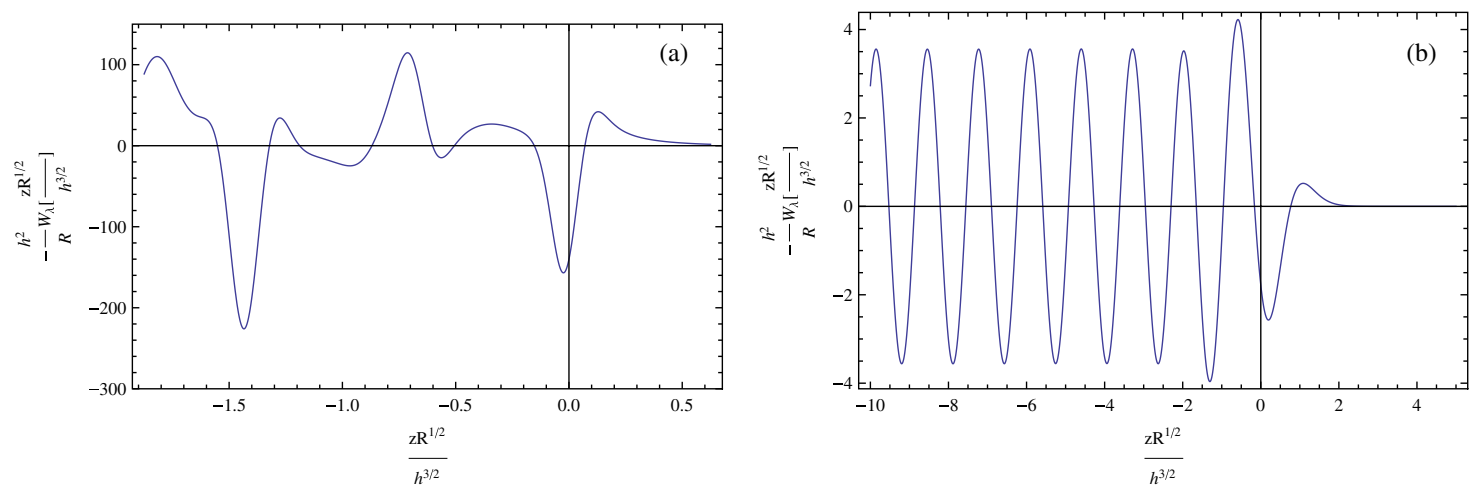

FIG. 5 (color online). Bunch wake as a function of the dimensionless position $\hat{z}$ for (a) $\chi=0.0625$ and (b) $\chi=0.5$ when a single mode is dominant. Note that there is a factor of 20 reduction resulting from the larger shielding parameter.

The bunch wake of two different shielding parameters $\chi=0.0625$ and $\chi=0.5$ are plotted to show the effect of shielding from the square chamber. These two particular values are chosen to compare with the steady wakes computed by Stupakov and Kotelnikov [19] using the mode expansion method under the paraxial approximation. Our results shown in Fig. 5 agree perfectly with theirs as shown in the (a) and (c) plots of Fig. 4 in their paper. Moreover, our results agree with the steady wake computed numerically by solving the parabolic equation [17].

In reality, the bunch will be dynamically deformed away from a Gaussian by the wakefield. We continue to investigate the longitudinal collective instability driven by the point-charge wake.

\section{VLASOV-FOKKER-PLANCK EQUATION}

Without any collective force, the beam distribution reaches a Gaussian equilibrium characterized by its length $\sigma_{z}$ and relative energy spread $\sigma_{\delta}$ in several damping times $\tau_{d}$ in electron storage rings. The energy spread $\sigma_{\delta}$ is largely determined by the ring geometry and the beam energy and the bunch length $\sigma_{z}$ is given by [23]

$$
\sigma_{z}=\alpha c \sigma_{\delta} / \omega_{s}
$$

where $\alpha$ is the momentum compaction factor and $\omega_{s}$ the angular synchrotron frequency.

With collective force, it is convenient to introduce a normalized coordinate system: $\hat{q}=z / \sigma_{z}$, where $z$ is the longitudinal position with the positive $z$ axis pointing to the front; $\hat{p}=-\delta / \sigma_{\delta}$, where $\delta$ is the relative energy deviation. When there is a collective force induced by the bunch distribution $\lambda(\hat{q}, \hat{\theta})$ through the wakefield $W(\hat{q})$, the evolution of the beam density distribution $\psi(\hat{q}, \hat{p} ; \hat{\theta})$ is governed by the Vlasov-Fokker-Planck (VFP) equation [24],

$$
\frac{\partial \psi}{\partial \hat{\theta}}-\{H, \psi\}=2 \hat{\beta} \frac{\partial}{\partial \hat{p}}\left(\hat{p} \psi+\frac{\partial \psi}{\partial \hat{p}}\right),
$$

where the Poisson bracket is defined by $\{f, g\}=$ $\frac{\partial f}{\partial \hat{q}} \frac{\partial g}{\partial \hat{p}}-\frac{\partial g}{\partial \hat{q}} \frac{\partial f}{\partial \hat{p}}$. The Hamiltonian

$$
\begin{aligned}
H(\hat{q}, \hat{p} ; \hat{\theta})= & \frac{1}{2}\left(\hat{q}^{2}+\hat{p}^{2}\right)-\hat{I} \int_{-\infty}^{\hat{q}} d \hat{q}^{\prime \prime} \\
& \times \int_{-\infty}^{\infty} d \hat{q}^{\prime} \lambda\left(\hat{q}^{\prime}, \hat{\theta}\right) W\left(\hat{q}^{\prime \prime}-\hat{q}^{\prime}\right),
\end{aligned}
$$

with $\lambda(\hat{q}, \hat{\theta})=\int_{-\infty}^{\infty} \psi(\hat{q}, \hat{p} ; \hat{\theta}) d \hat{p} \quad$ and normalizes to $\int_{-\infty}^{\infty} \lambda(\hat{q}, \hat{\theta}) d \hat{q}=1$. The independent variable $\hat{\theta}=\omega_{s} t$ with $t$ the time and $\hat{\beta}=1 / \omega_{s} \tau_{d}$. We define normalized current as [25]

$$
\hat{I}=\frac{r_{e} N_{b}}{2 \pi \nu_{s} \gamma \sigma_{\delta}}
$$

with $r_{e}$ is the classical electron radius, $\gamma$ is the Lorentz factor, $\nu_{s}$ is the synchrotron tune, and $N_{b}$ is the bunch population. In practice, it is useful to rewrite $\hat{I}$ in terms of the bunch current $I_{b}$ using Eq. (7.1),

$$
\hat{I}=\frac{\sigma_{z} I_{b}}{\alpha \gamma \sigma_{\delta}^{2} I_{A}},
$$

where $I_{A}=m c^{3} / e=17045 \mathrm{~A}$ is the Alfvén current. $\hat{I}$ has dimension of length, which cancels the dimension of the wakefield,

$$
\begin{aligned}
W(\hat{q})= & \frac{R}{h^{2}} \sum_{j} \hat{k}_{j} \frac{R_{s}}{Q}\left(\hat{k}_{j}\right)\left[\Theta(-\hat{q}) \cos \left(\hat{k}_{j} \chi \hat{q}\right)\right. \\
& \left.-\frac{\operatorname{sgn}(\hat{q})}{2} e^{-\hat{k}_{j} \chi|\hat{q}|}\right] .
\end{aligned}
$$

Here we have used Eq. (5.13) for the point-charge wake and $\hat{z}=\chi \hat{q}$. It is more convenient to absorb the dimension in the wake to a dimensionless current, 


$$
\xi=\frac{\hat{I} R^{1 / 3}}{\sigma_{z}^{4 / 3}},
$$

along with the dimensionless wake

$$
\begin{aligned}
W_{\xi}(\hat{q})= & \chi^{4 / 3} \sum_{j} \hat{k}_{j} \frac{R_{s}}{Q}\left(\hat{k}_{j}\right)\left[\Theta(-\hat{q}) \cos \left(\hat{k}_{j} \chi \hat{q}\right)\right. \\
& \left.-\frac{\operatorname{sgn}(\hat{q})}{2} e^{-\hat{k}_{j} \chi|\hat{q}|}\right]
\end{aligned}
$$

It is worth noting that this wake depends only on the shielding parameter $\chi$ and the aspect ratio $A$. The Hamiltonian becomes

$$
\begin{aligned}
H(\hat{q}, \hat{p} ; \hat{\theta})= & \frac{1}{2}\left(\hat{q}^{2}+\hat{p}^{2}\right)-\xi \int_{-\infty}^{\hat{q}} d \hat{q}^{\prime \prime} \\
& \times \int_{-\infty}^{\infty} d \hat{q}^{\prime} \lambda\left(\hat{q}^{\prime}, \hat{\theta}\right) W_{\xi}\left(\hat{q}^{\prime \prime}-\hat{q}^{\prime}\right) .
\end{aligned}
$$

If there is a threshold of collective instability that is described by the VFP equation, then it is obvious that the threshold should be given by

$$
\xi=\xi^{\mathrm{th}}(\chi, A, \hat{\beta}),
$$

where $\xi^{\text {th }}$ is a function we shall determine. For the parallel plates, we have found $[15,16]$

$$
\xi^{\text {th }}(\chi, \infty, 0)=0.5+0.34 \chi .
$$

Note that the coefficient 0.34 , seemingly differing from 0.12 in [15], is due to a different definition of the height. Aside from a dip at $\chi=0.25$, the instability grows sufficiently fast so that the dependence of threshold on the damping rate $\hat{\beta}$ is negligible.

\section{SIMULATION}

It is easy to see that the VFP equation has a static solution in form of

$$
\psi(\hat{q}, \hat{p})=\frac{1}{\kappa \sqrt{2 \pi}} \exp (-H)=\lambda(\hat{q}) \exp \left(-\frac{\hat{p}^{2}}{2}\right) / \sqrt{2 \pi},
$$

which leads to the well-known Haissinski equation [26],

$$
\begin{aligned}
\lambda(\hat{q})= & \frac{1}{\kappa} \exp \left[-\frac{\hat{q}^{2}}{2}+\xi \int_{-\infty}^{\hat{q}} d \hat{q}^{\prime \prime}\right. \\
& \left.\times \int_{-\infty}^{\infty} d \hat{q}^{\prime} \lambda\left(\hat{q}^{\prime}\right) W_{\xi}\left(\hat{q}^{\prime \prime}-\hat{q}^{\prime}\right)\right],
\end{aligned}
$$

where the constant $\kappa$ is necessary for the normalization. In general, this nonlinear integral equation can be solved numerically using Newton's iteration starting from a Gaussian distribution. We know that the Haissinski distribution is not only a static solution but also the equilibrium distribution of the VFP equation at sufficiently low current. Above a threshold of $\xi$, the Haissinski distribution becomes unstable. The associated instability is commonly referred to as the microwave instability. In practice, we always start with Haissinski distribution and then detect the threshold by simply monitoring any deviation of $\sigma_{\hat{p}}$ away from 1.

In numerical simulation, we continue to use a robust algorithm developed by Warnock and Ellison [27] to solve the VFP equation on grid points on a rectangular mesh. Reliable results require a mesh size comparable to the smallest length scale of the longitudinal motion. Given the computing power of a Linux computer, that limits the shielding parameter $\chi \leq 1$ for a mesh of $2400 \times 2400$ that covers a maximum of eight sigma of beam size. For a typical run, we set the damping rate at $\hat{\beta}=5 \times 10^{-4}$ and use 10240 steps per synchrotron period running up to 200 periods.

We have performed stability calculations for aspect ratios of $A=1,2$ and 3. Our results of the threshold function $\xi^{\text {th }}$ are plotted in Fig. 6. At a higher end of the shielding parameters, $0.3<\chi \leq 1$, there is at least a factor of 2 in reduction of the threshold for a square chamber in comparison to that of the parallel plates. For a rectangular chamber with sufficiently large aspect ratio $A \geq 2$, there is not too much difference. At the lower end, $0<\chi \leq 0.3$, all the thresholds are low, similar to the dip [15] seen in the parallel-plate model. The range of the shielding parameters we have studied here covers the bunch length $\sigma_{z}$ close to $1 \mathrm{~mm}$ which is necessary to generate sufficient $\mathrm{THz}$ radiation in the third-generation light sources.

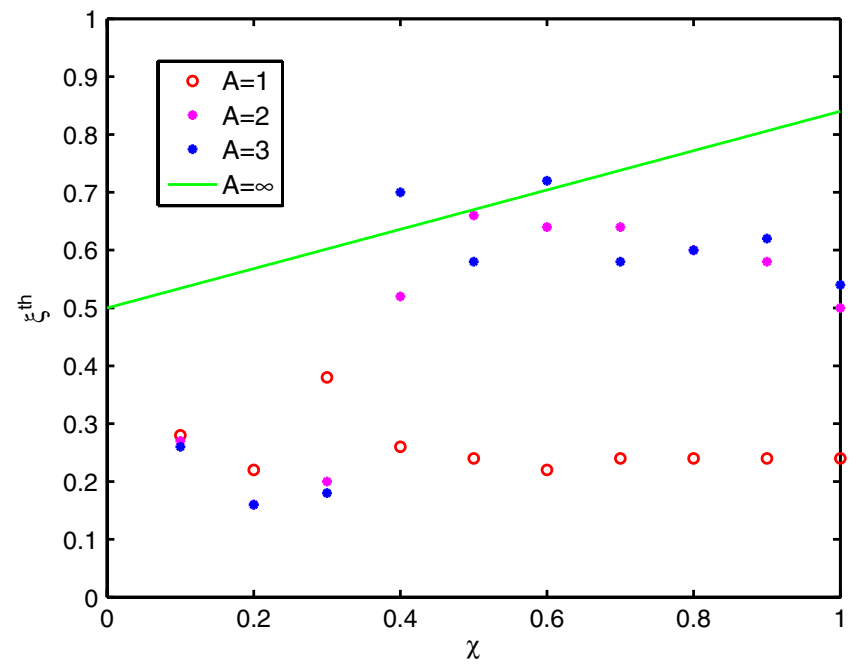

FIG. 6 (color online). Simulation results of the threshold as a function of the shielding parameter for rectangular chambers with various aspect ratios. The solid line represents $\xi^{\text {th }}=0.5+0.34 \chi$ for the parallel plate model. 


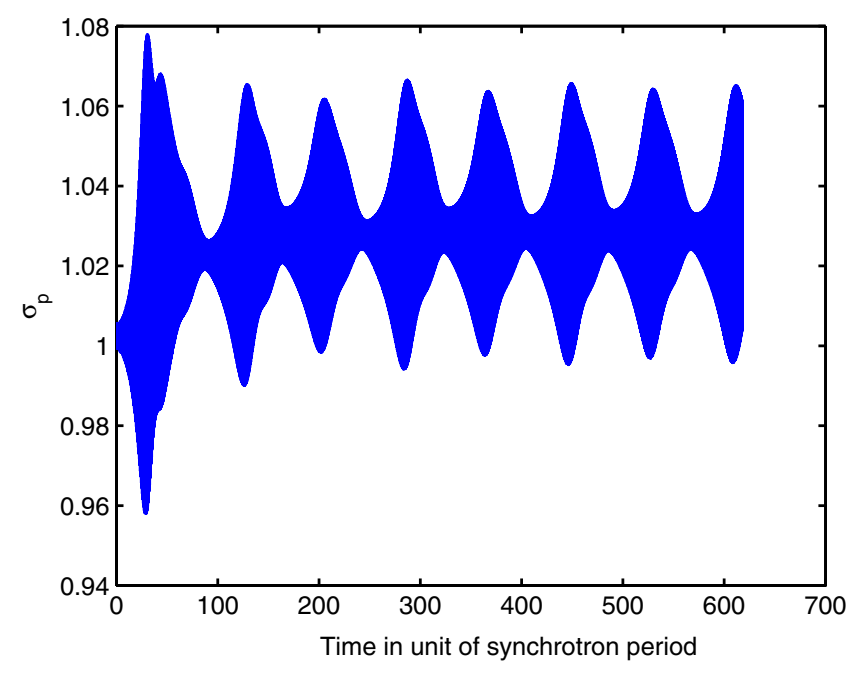

FIG. 7 (color online). A sawtooth instability seen at the dimensionless current $\xi=0.38$ and the shielding parameter $\chi=0.3$ in a square vacuum chamber. It has an approximate period of 80 synchrotron oscillations or $4 \%$ of the damping time.

Obviously, there is much more work that is necessary to obtain the threshold function $\xi^{\text {th }}(\chi, A, \hat{\beta})$. Given a highly oscillatory nature of the wake, this work may require the power of a supercomputer running a parallel code, especially for large shielding parameter $\chi$.

Beyond the threshold, there are various ways that the instability can manifest itself. Among them, the most fascinating one is shown in Fig. 7, behaving like the sawtooth instability. Zooming in, we see a much faster oscillation near frequency of $2 \omega_{s}$ bounded by the envelope. This periodical feature resembles the bursting observations $[6,7]$ from the THz radiation. Here this kind of phenomena occurs at the region where the weak instability was observed [15].

\section{CONCLUSION}

The simple scaling law of the threshold found in the parallel-plate model can be extended to the case of a rectangular vacuum chamber. In addition, the threshold depends on the aspect ratio of the chamber. The validity of the scaling law is relying on the conditions of $\sigma_{z} \ll R$, $h \ll R$, and $w \ll R$. Clearly, these conditions are valid for most electron storage rings. As a result, the scaling law is an excellent approximation to parametrize the longitudinal instability driven by CSR. Moreover, one should avoid using a square chamber in an electron storage ring because its sidewalls seem too close for CSR. With the same height, a rectangular chamber with aspect ratio $2: 1$ should be a better choice.

Given the threshold $\xi^{\text {th }}$, the threshold of bunch current $I_{b}^{\text {th }}$ can be written as [16]

$$
I_{b}^{\text {th }}=\frac{8 \pi^{2} \xi^{\text {th }} \sigma_{z}^{7 / 3} V_{\mathrm{rf}} f_{\mathrm{rf}} f_{\mathrm{rev}} \cos \phi_{s}}{c^{2} Z_{0} R^{1 / 3}},
$$

where $V_{\text {rf }}$ and $f_{\text {rf }}$ are the voltage and frequency of the $\mathrm{rf}$ cavities, $f_{\text {rev }}$ the revolution frequency, $\phi_{s}$ the synchronous phase, and $Z_{0}=120 \pi \Omega$ impedance in free space. It shows that there is a simple but extremely unfavorable scaling law against the bunch length $\sigma_{z}$. This scaling behavior was universally observed in the electron storage rings throughout the world. It is worth mentioning that a deviation from this scaling should be expected but can be explained by the dependence of $\xi^{\text {th }}$ on the shielding parameter $\chi$.

Finally, to finish our paper on a positive note, we shall emphasize that increasing longitudinal focusing by the rf, namely the quantity of $V_{\mathrm{rf}} f_{\mathrm{rf}}$, is an effective path to reduce the bunch length while maintaining the beam current. This scheme requires us to introduce many superconducting rf's at a higher frequency in future storage rings.

\section{ACKNOWLEDGMENTS}

I would like to thank my colleagues: Karl Bane, Alex Chao, Gennady Stupakov, and especially Robert Warnock for many helpful discussions and sharing their insights. This work was supported by the U.S. Department of Energy under Contract No. DE-AC02-76SF00515.

[1] J. Schwinger, LBL Report No. LBL-39088, 1996 (unpublished).

[2] J. Nodvick and D. Saxon, Phys. Rev. 96, 180 (1954).

[3] R. L. Warnock and P. Morton, Report No. SLAC-PUB5462, 1988.

[4] J. B. Murphy, S. Krinsky, and R. L. Gluckstern, Part. Accel. 57, 9 (1997).

[5] G. L. Carr, S. L. Kramer, J. B. Murphy, R. P. S. M. Lobo, and D. B. Tanner, Nucl. Instrum. Methods Phys. Res., Sect. A 463, 387 (2001).

[6] M. Abo-Bakr, J. Feikes, K. Holldack, G. Wustefeld, and H.-W Hubers, Phys. Rev. Lett. 88, 254801 (2002).

[7] J. Byrd, W. Leemans, A. Loftsdottir, B. Marcelis, M. Martin, W. McKinney, F. Sannibale, T. Scarvie, and C. Steier, Phys. Rev. Lett. 89, 224801 (2002).

[8] F. Sannibale et al., Phys. Rev. Lett. 93, 094801 (2004).

[9] G. Stupakov and S. Heifets, Phys. Rev. ST Accel. Beams 5, 054402 (2002).

[10] M. Venturini and R. Warnock, Phys. Rev. Lett. 89, 224802 (2002).

[11] G. Wustefeld et al., in Proceedings of the International Particle Accelerator Conference, Kyoto, Japan (ICR, Kyoto, 2010), p. 2508.

[12] P. Kuske, in Proceedings of the 23rd Particle Accelerator Conference, Vancouver, Canada, 2009 (IEEE, Piscataway, NJ, 2009), p. 4682.

[13] M. Klein et al., in Proceedings of the 23rd Particle Accelerator Conference, Vancouver, Canada, 2009 (IEEE, Piscataway, NJ, 2009), p. 4761. 
[14] M. Ries et al., in Proceedings of the 3rd International Particle Accelerator Conference, New Orleans, Louisiana, USA, 2012 (IEEE, Piscataway, NJ, 2012), p. 3030.

[15] K. L. F. Bane, Y. Cai, and G. Stupakov, Phys. Rev. ST Accel. Beams 13, 104402 (2010).

[16] Yunhai Cai, in Proceedings of the 2nd International Particle Accelerator Conference, San Sebastián, Spain (EPS-AG, Spain, 2011), p. 3774.

[17] T. Agoh and K. Yokoya, Phys. Rev. ST Accel. Beams 7, 054403 (2004).

[18] Tomonori Agoh, Phys. Rev. ST Accel. Beams 12, 094402 (2009).

[19] G. V. Stupakov and I. A. Kotelnikov, Phys. Rev. ST Accel. Beams 12, 104401 (2009).
[20] P. M. Morse and H. Feshbach, Methods of Theoretical Physics, Part I (McGraw-Hill, New York, 1953).

[21] A. W. Chao, Physics of Collective Beam Instabilities in High Energy Accelerators (John Wiley \& Sons, New York, 1993).

[22] F. W. J. Olver, Asymptotics and Special Functions (Academic Press, New York, 1974).

[23] M. Sands, SLAC Report No. 121, 1979.

[24] Henri Bruck, Circular Particle Accelerator (Los Alamos Scientific Laboratory, Los Alamos, NM, 1972).

[25] K. Oide and K. Yokoya, KEK Report No. 90-10, 1990.

[26] J. Haissinski, Nuovo Cimento B 18, 72 (1973).

[27] R. L. Warnock and J. A. Ellison, in The Physics of High Brightness Beams (World Scientific, Singapore, 2000), pp. 322-348. 